\title{
Comparison of Tooth Shade Colour Recorded Using Digital Single Lens Reflex Camera and Mobile Cameras with Manually Adjusted Parameters
}

\author{
Vidya S. Bhat ${ }^{1}$, Sanath Kumar Shetty², Khizer Ishaquddin Syed ${ }^{3}$, Sanha Razdan ${ }^{4}$ \\ 1,2,3,4 Department of Prosthodontics, Yenepoya Dental College, Deralakatte, Mangalore, Karnataka, India.
}

\section{ABSTRACT}

\section{BACKGROUND}

Visual selection by using shade guides is the most common but also the most subjective technique, influenced by factors such as the age of the observer and eye fatigue. Digital imaging provides a practical and consistent method of determining tooth colour. A suitable alternative for DSLR cameras may be a mobile phone camera. Recent smart phones have manual control which helps to manually adjust parameters. Female patients usually use lipstick, which may alter the tooth colour perception, but there is no clinical evidence showing the effect of lipstick on tooth shade. The purpose of this study was to evaluate the reliability of using phone camera and the effect of lipstick on the shade of the tooth.

\section{METHODS}

This study was conducted on 11 female individuals within the age of 18 - 25 years. Eight photographs were made of all 11 participants, using 3 different smart phone cameras and Nikon D3400 DSLR camera with twin flash and cross-polaroid filter was used as the control group. The parameters of all the cameras were standardized for every image. All digital photographs were evaluated with a Digital Colour Meter software, which gave the parameters in the RGB colour codes and CIE L, a*b* colour space.

\section{RESULTS}

There was no statistically significant difference in the $\mathrm{L}^{*}$ and $\mathrm{R}^{*}$ values between the control and the smartphone camera groups. There was no statistically significant difference in $\mathrm{L}^{*}$ and $\mathrm{R}^{*}$ values before and after applying lipstick in all the groups. There was no significant difference in $\mathrm{L}^{*}$ and $\mathrm{R}^{*}$ values in all the groups before and after applying lipstick.

\section{CONCLUSIONS}

Recent smartphone cameras with manually adjusted parameters including unprocessed or RAW images can be used as a reliable tool for shade matching. Shade matching can be done even after applying lipstick, as there was no difference seen in this study.

\section{KEY WORDS}

Esthetics, Shade, Lipstick, Spectophotometer, Shade
Corresponding Author: Dr. Khizer Ishaquddin Syed Department of Prosthodontics \& Crown and Bridge, Yenepoya Dental College, Yenepoya University, University Road, Deralakatte, Mangalore - 575018,

Karnataka, India.

E-mail: khizerronaldo9@gmail.com

DOI: $10.14260 /$ jemds/2021/528

How to Cite This Article:

Bhat VS, Shetty SK, Syed KI et al. Comparison of tooth shade colour recorded using digital single lens reflex camera and mobile cameras with manually adjusted parameters. J Evolution Med Dent Sci 2021;10(32):2577-2581, DOI: $10.14260 /$ jemds/2021/528

Submission 11-04-2021,

Peer Review 08-07-2021,

Acceptance 14-07-2021,

Published 09-08-2021.

Copyright (C) 2021 Vidya S. Bhat et al. This is an open access article distributed under Creative Commons Attribution License [Attribution 4.0 International (CC BY 4.0)] 


\section{BACKGROUND}

Colour matching in dentistry is considered a subjective process, dependent on 3 main factors: light source, object, and observer. Although no single method is considered standard, 2 methods exist to evaluate colour in dentistry: visual and instrumental. ${ }^{1}$ Visual selection by using shade guides is the most common but also the most subjective technique, influenced by factors such as the age of the observer and eye fatigue. ${ }^{2}$ Therefore, instrumental methods such as spectrometers and colourimeters have gained attention. However, they have poor interdevice reliability and require specific and expensive technology which is not always available to the clinician. Digital cameras are a common communication tool in dental offices and can be used for electronic shade registration, although they still present a certain degree of subjectivity in shade selection. ${ }^{3}$ Digital imaging provides a practical and consistent method of determining tooth colour and transmitting this information to the dental laboratory technician. Adding a cross-polarized light filter has been suggested to improve accuracy by eliminating the brightness of the environment and the specular reflection produced from saliva or teeth. ${ }^{4}$ DSLR camera besides offering great picture quality, allows to manually controlling the exposure of a shot. A suitable alternative for DSLR cameras may be a mobile phone camera. Mobile phones are easily accessible and handy to carry around. Recent smart phones have manual control which helps to manually adjust parameters like shutter speed, ISO, white balance etc and some phones even capture an unprocessed or RAW image just like DSLRs. ${ }^{5}$ Female patients usually use lipstick, which may alter the tooth colour perception, but there is no clinical evidence showing the effect of lipstick on tooth shade. ${ }^{6-10}$ The purpose of this study was to evaluate the reliability of using phone camera and the effect of lipstick on the shade of the tooth.

\section{METHODS}

An experimental study was conducted on 11 females in the age group of 18 - 25 years at Yenepoya Dental College, Mangalore, India.

\section{Study Duration}

Topic selection and review of literature: September 2020 Protocol preparation and ethical approval: October 2020 Data collection and data entry: November 2020

Data analysis: November 2020

Final report and submission: December 2020

\section{Inclusion Criteria}

Age group 18 - 25 years

All anterior natural teeth should be present.

\section{Exclusion Criteria}

Teeth bleaching within 6 months
Anterior teeth with restoration

Anterior teeth with crown or bridges

\section{Ethical Statement}

Ethical approval was obtained from the Yenepoya Ethical Committee. Written informed consent was obtained from all the participants and they were assured of confidentiality.

Eight photographs were made of all 11 participants $(\mathrm{N}=$ 88), using 3 different smart phone cameras (Huawei P9 Pro, Samsung M31s, One Plus 7T)and Nikon D3400 DSLR camera with twin flash and cross-polaroid filter. The DSLR camera was used as the control group. The parameters of all the cameras were standardized for every image in the following values: ISO 200, f25, shutter speed 1/125, white balance flash, and image quality in RAW format. The camera distance was set in a range of $30-50 \mathrm{~mm}$ that allowed the teeth being well captured inside the image. To avoid tooth dehydration, participants were asked to close their mouths between photographs. The photographs of each participant were taken with Nikon camera and smart phones. Then, they were asked to apply 2 coats of lipstick (Lakme Enrich Matte Lipstick WM13) and photos were taken again with the same standard procedure.

All digital photographs were evaluated with a software (Digital Colour Meter version 5.1 for MacOS; Apple Inc.), which gave the parameters in the RGB colour codes and CIEL,a*b*colour space.

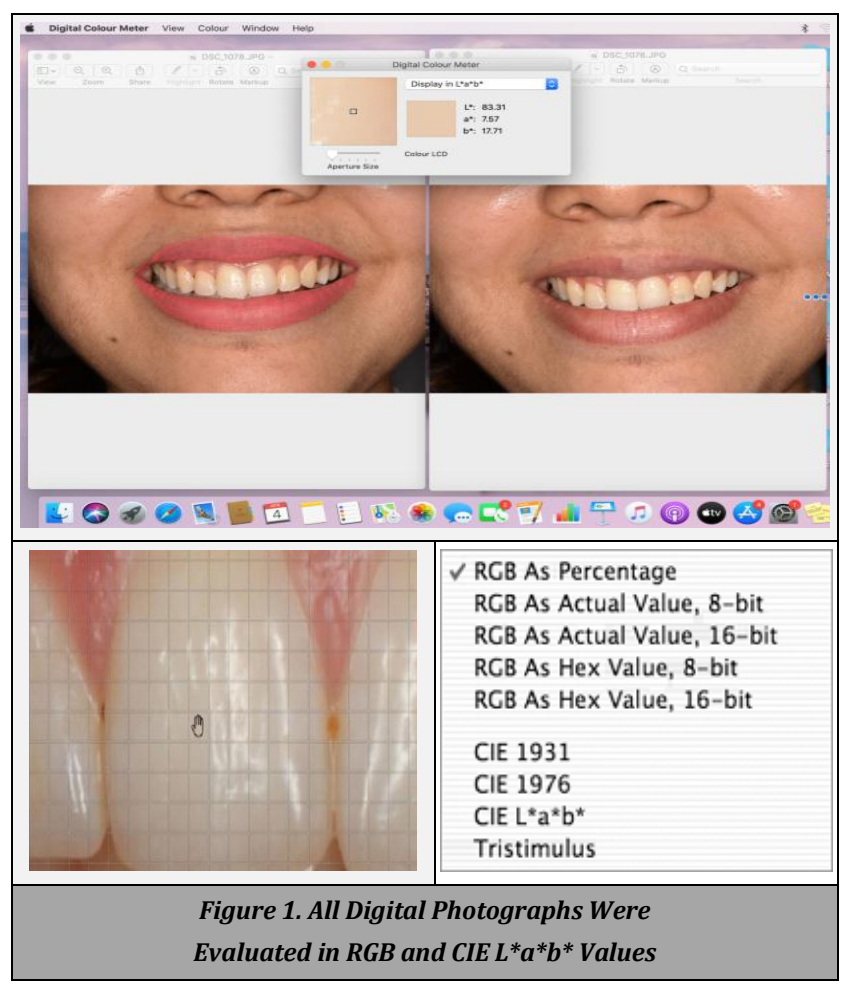

Colour measurements were made in the center of the maxillary right central incisor, with all photographs at the same position; the colour window was adjusted to the maximum size possible, and the CIEL, a*b* coordinates were measured.

Subsequently, values for luminosity from three groups of digital image equipment were statistically analysed by using generalized estimating equations (GEE) together with the 
Huber-White standard error estimates to identify differences among the methods, independently for use or not of the lipstick ( $\mathrm{N}=8$ per group).

\section{Statistical Analysis}

All the data was collected in Excel sheet and for analysis SPSS version 23 was used. Descriptive statistics was used to find out mean and standard deviation of each group. One-way ANOVA was used for comparison of 4 groups. $P$ value less than 0.001 was considered as significant. $(\mathrm{P}<\alpha ; \alpha=0.01)$

\section{RESULTS}

The mean age of participants was 23 years. (SD 1.12) There was no statistically significant difference in the $\mathrm{L}^{*}$ and $\mathrm{R}^{*}$ values between the control and the smartphone camera groups. (Table 1, 2, 3, 4)

There was no statistically significant difference in $\mathrm{L}^{*}(\mathrm{P}-$ value: 0.857 ) (Table 1 ) and $\mathrm{R}^{*}$ (P-value: $0.046^{*}$ ) (Table 2 ) values before applying lipstick in all the groups. There was no statistically significant difference in $\mathrm{L}^{*}$ (P-value: 0.962) (Table 3) and $\mathrm{R}^{*}(\mathrm{P}$-value: 0.276$)$ values after applying lipstick.

There was no significant difference in $\mathrm{L}^{*}$ (P-value: 0.106$)$ and $\mathrm{R}^{*}$ (P-value: 0.631 ) values in all the groups before and after applying lipstick.

\begin{tabular}{|cccccc|}
\hline Colour Codes & Camera & Mean & Std. Deviation & F Statistic P-Value \\
L* $^{*}$ & DSLR & 83.3118 & 3.12641 & & \\
& Huawei & 82.8918 & 4.45374 & & \\
& J5 & 82.3209 & 4.80388 & 0.255 & 0.857 \\
& one+ & 83.7282 & 3.11159 & & \\
R $^{*}$ & Total & 83.0632 & 3.84473 & & \\
& DSLR & 237.64 & 6.816 & & \\
& Huawei & 226.36 & 11.360 & 2.916 & $0.046^{*}$ \\
& J5 & 227.55 & 12.501 & & \\
\hline & one+ & 232.73 & 8.533 & & \\
Total & $\mathbf{2 3 1 . 0 7}$ & $\mathbf{1 0 . 7 0 8}$ & & \\
\hline
\end{tabular}

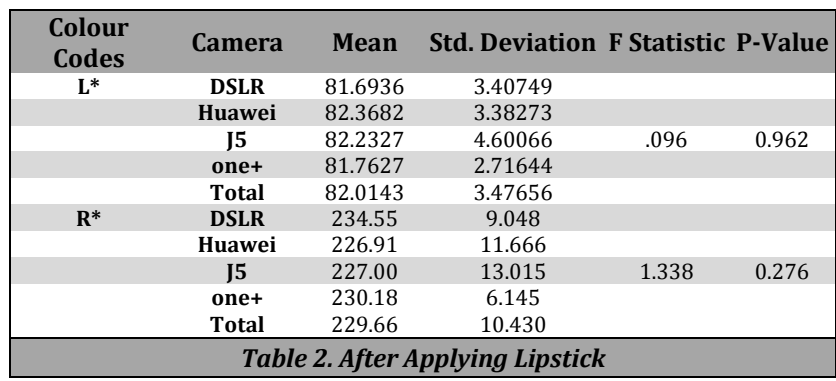

\begin{tabular}{|c|c|c|c|c|c|}
\hline $\begin{array}{c}\text { Colour } \\
\text { Codes }\end{array}$ & Lipstick & Mean & Std. Deviation & F Statistic & P-Value \\
\hline \multirow[t]{2}{*}{$\mathbf{L}^{*}$} & Without lipstick & 83.3118 & 3.12641 & 2.874 & 0.376 \\
\hline & With lipstick & 81.6936 & 3.40749 & & \\
\hline \multirow[t]{2}{*}{$\mathbf{R}^{*}$} & Without lipstick & 237.6364 & 6.81576 & 0.865 & 0.259 \\
\hline & With lipstick & 234.5455 & 9.04835 & & \\
\hline
\end{tabular}

\section{DISCUSSION}

Shade selection in dental practice is an important but a difficult task.11,12 Although there are many kinds of shade guides available in the market, it is still not easy to match shade tabs with teeth intra-orally. A clinical study reported between $44 \%$ and $63 \%$ colour mismatch in ceramic restorations because of factors such as the colour-measuring device used. ${ }^{3}$

Visual selection by using shade guides is the most common but also the most subjective technique. Instrumental methods for reducing the problems of objectivity in colour registration have been developed, including spectrophotometers, colourimeters, and digital cameras. ${ }^{13}$ Compared with colour match by the human eye (conventional techniques), spectrophotometers offered a $33 \%$ increase in accuracy and a more objective match in $93.3 \%$ of selections. ${ }^{14,15}$ A combination of visual and instrumental techniques has been recommended for better perceptibility and acceptability. ${ }^{13}$ However, disadvantages are increased costs and the need for training. ${ }^{3}$

The uses of intra-oral spectrophotometers may fall into a shortcoming that the small (usually $3 \mathrm{~mm}$ ) window of the devices may not capture reliably the real colour information of the whole tooth surface. It poses difficulties in correctly positioning the device over the tooth surface during the measuring procedure, the improper measurement of the curved translucent surfaces found on teeth using the contact type instruments that are designed for flat surfaces, ${ }^{16}$ and these equipments employ mean values or intensity histograms for representing the shade zone for colour labelling and ignore the geometric distribution of colour within the region.

Digital cameras are a common communication tool in dental offices and can be used for electronic shade registration. Digital photographs can be easily stored and transmitted to a technician, providing good quality information regarding colour across the tooth surface, shape, and characteristic features. ${ }^{17}$ In this study, Nikon D3400 was used and its reliability for intraoral shade evaluation was seen in previous studies.

In previous studies, smart phone and DSLR cameras were used for shade evaluation, and the results showed that the DSLR cameras were far superior because the phones used in those studies did not have manually adjusted parameters like shutter speed, ISO, white balance and RAW image quality. ${ }^{2,18}$ The spectrophotometric analysis of the images was done using Digital Colour Meter (version 5.1 for MacOS; Apple Inc.)

Manual mode on a camera determines the exposure of an image by selecting an aperture value and a shutter speed value. ISO values ranges from 100 to 3200 . Higher ISOs are best used in lower light, while lower ISOs are better for brightly lit interiors or full sun outdoors. ${ }^{5} \mathrm{~A}$ standard speed to start off with would be ISO 200.The aperture consists of small blades in the lens that creates an adjustable, octagonal shape. It tells you how big the hole is present in the lens. The smaller the hole, lesser the light passes through it. The wider the hole is, the more light will enter the lens. The aperture is measured by f-stop. The higher the f-stop (f22), the smaller the hole and the darker the image will be. The f-stop also measures the depth of field. A lower f-stop (f2.8) will give a more depth to the image. The shutter speed tells the amount of time the camera will be exposed to light. ${ }^{19}$ The duration of time is shown in a fraction of seconds, for example it could be $1 / 2$ second or $1 / 200$ second. A speed of $1 / 200$ second is faster than $1 / 2$ second. Using a faster speed means the image will be darker. If you want to lighten the image, use 
a slower shutter speed. However, a faster shutter speed will catch a frame of movement. ${ }^{20}$

In raw images, the file preserves the information captured at the time of exposure. The purpose of raw image formats is to save, with minimum loss of information, data obtained from the sensor. The same image can be edited in a variety of different ways without affecting image quality. ${ }^{21}$

Digital colour meter is an application or software for measuring and displaying the colour values of pixels displayed on the screen of a computer.The utility presents a window onto the screen which includes a cursor which by default is $1 \times 1$ pixel in size. The colour displayed in that pixel is shown as a colour value which may be represented as decimal or hexadecimal RGB triplets, CIE 1931, CIE 1976 or CIELAB triplets. The displayed colour could be copied either as a solid colour or as the colour value which represents it, to be used in other applications. ${ }^{22}$

The results of this study stated that, there was no statistically significant difference between the RGB and CIE $\mathrm{L}, \mathrm{a}^{*} \mathrm{~b}^{*}$ values in the smartphone and the control group, as the cameras in every group had standardised the manually adjusted parameters and RAW images. CIE L,a*b* and RGB values were evaluated in this study, $L^{*}$ was used because that is the parameter that was more variable concerning colours, whereas $a^{*}$ and $b^{*}$ values were more standardized. Whereas in RGB colour coding, $\mathrm{R}^{*}$ was the parameter which was used for analysis.

RGB values showed better results although no statistically significant difference was noted. There are no studies relating to the shade evaluation of teeth before and after applying lipstick, as it was stated that shade evaluation should not be done after applying lipstick. ${ }^{6-14}$ The results of this study concluded that there is no statistically significant difference before and after applying lipstick and shade evaluation can be done even if the lipstick is applied.

According to the American Dental Association (ADA), for measuring colour differences of dental shades, the formula was given as ${ }^{23}: \Delta \mathrm{E}=\left(\left(\mathrm{L}^{*}{ }_{\mathrm{i}}-\mathrm{L}_{\mathrm{j}}\right)^{2}+\left(\mathrm{a}_{\mathrm{i}}{ }_{\mathrm{i}}-\mathrm{a}_{\mathrm{j}}\right)^{2}+\left(\mathrm{b}_{\mathrm{i}} \mathrm{i} \mathrm{b}_{\mathrm{j}}\right)^{2}\right)^{1 / 2}$

Clinical observations have claimed that a $\Delta \mathrm{E}$ value greater than 3.7 is graded as a poor match in dental shades. Johnston and $\mathrm{Kao}^{24}$ reported in a clinical study that $\Delta \mathrm{E} 6.85$ was the average colour difference between compared teeth rated as mismatch within the normal range of tooth colour in the oral environment. Further studies can be done on a larger population; evaluation can be done using shade tabs and can be evaluated in different environment.

\section{CONCLUSIONS}

Recent smartphone cameras with manually adjusted parameters including unprocessed or RAW images can be used as a reliable tool for shade matching. Evaluation of shade can be done accurately by capturing images of teeth with the shade tabs and calibrating on the Digital Colour Meter software. Shade matching can be done even after applying lipstick, as there was no difference seen in this study.

Data sharing statement provided by the authors is available with the full text of this article at jemds.com.

Financial or other competing interests: None.
Disclosure forms provided by the authors are available with the full text of this article at jemds.com.

\section{REFERENCES}

[1] Hardan L. Mobile dental photography (MDP): a new era in dental documentation. International Arab Journal of Dentistry 2017;8(3):115-8.

[2] Sampaio CS, Atria PJ, Hirata R, et al. Variability of colour matching with different digital photography techniques and a gray reference card. The Journal of Prosthetic Dentistry 2019;121(2):333-9.

[3] Mahn E, Tortora SC, Olate B, et al. Comparison of visual analog shade matching, a digital visual method with a cross-polarized light filter and a spectrophotometer for dental colour matching. The Journal of Prosthetic Dentistry 2021;125(3):511-16.

[4] Tam WK, Lee HJ. Dental shade matching using a digital camera. Journal of Dentistry 2012;40(Suppl 2):e3-10.

[5] Azhar NAM, Ahmad A, Samad A, et al. Comparative geometric and radiometric evaluation of mobile phone, compact and DSLR cameras. In: 2013 IEEE 9th International Colloquium on Signal Processing and its Applications 2013: p. 353-8.

[6] Bona DA, Barrett AA, Rosa V, et al. Visual and instrumental agreement in dental shade selection: three distinct observer populations and shade matching protocols. Dental Materials 2009;25(2):276-81.

[7] Li Q, Wang YN. Comparison of shade matching by visual observation and an intraoral dental colourimeter. Journal of Oral Rehabilitation 2007;34(11):848-54.

[8] Al-Saleh S, Labban M, Al-Hariri M, et al. Evaluation of self shade matching ability of dental students using visual and instrumental means. Journal of Dentistry 2012;40(Suppl 1):e82-7.

[9] $\mathrm{Li} \mathrm{Q}, \mathrm{Yu} \mathrm{H}$, Wang YN. In vivo spectroradiometric evaluation of colour matching errors among five shade guides. Journal of Oral Rehabilitation 2009;36(1):65-70.

[10] Sorensen JA, Torres TJ. Improved colour matching of metal-ceramic restorations. Part I: a systematic method for shade determination. The Journal of Prosthetic Dentistry 1987;58(2):133-9.

[11] Sproull RC. Colour matching in dentistry. Part III. Colour control. The Journal of Prosthetic Dentistry 1974;31(2):146-54.

[12] Preston JD. Current status of shade selection and colour matching. Quintessence Int 1985;16(1):47-58.

[13] Brewer JD, Wee A, Seghi R. Advances in colour matching. Dent Clin North Am 2004;48(2):v, 341-58.

[14] Chu SJ, Trushkowsky RD, Paravina RD. Dental colour matching instruments and systems. Review of clinical and research aspects. J Dent 2010;38(Suppl 2):e2-16.

[15] Rad IZ. In vivo evaluations of inter-observer reliability using VITA Easyshade advance 4.0 dental shadematching device. Acta Stomatol Croat 2016;50(1):34-9.

[16] Haywood VB, Leonard RH, Nelson CF, et al. Effectiveness, side effects and long-term status of nightguard vital bleaching. Journal of the American Dental Association 1994;125(9):1219-26. 
[17] Jarad FD, Russell MD, Moss BW. The use of digital imaging for colour matching and communication in restorative dentistry. Br Dent J 2005;199(1):43-9.

[18] Shrestha P, Poudel S. Comparison between mobile camera and DSLR camera photography for the evaluation of shade of anterior teeth - a cross-sectional study. Journal of Chitwan Medical College 2019;9(2):111-9.

[19] Javh J, Slavič J, Boltežar M. Experimental modal analysis on full-field DSLR camera footage using spectral optical flow imaging. Journal of Sound and Vibration 2018;434:213-20.
[20] Iyer VH. Dental photography-an image to improve the face of dental practice. An Off J of IDA 2019;6(2):52-7.

[21] Ahmad I. Digital dental photography. Part 1: an overview. British Dental Journal 2009;206(8):403-7.

[22] Ahmad I. Digital dental photography. Part 4: choosing a camera. British Dental Journal 2009;206(11):575-81.

[23] Douglas RD, Steinhauer T], Wee AG. Intraoral determination of the tolerance of dentists for perceptibility and acceptability of shade mismatch. J Prosthet Dent 2007;97(4):200-8.

[24] Johnston WM, Kao EC. Assessment of appearance match by visual observation and clinical colourimetry. Journal of Dental Research 1989;68(5):819-22. 